\title{
Variabilité génétique en croisement intra- et interpopulation dans deux populations de maïs fourrage
}

\author{
B. Gouesnard ${ }^{\star}$, A. Gallais et M. Lefort-Buson \\ INRA-CNRS-UPS, ferme du Moulon, 91190 Gif-sur-Yvette, France
}

(reçu le 27 janvier 1989; accepté le 5 juillet 1989)

\begin{abstract}
Résumé - A la suite d'un plan de croisement hiérarchique, la variabilité génétique révélée en croisement intra- et interpopulation dans 2 populations de maïs fourrage a été analysée aux cycles $C_{0}$ et $C_{1}$ de sélection. Les caractères étudiés sont les suivants : précocité de floraison, hauteur totale, hauteur de l'épi, longueur et largeur de la feuille de l'épi, rendement de la plante entière et teneur en matière sèche. La nature de la variabilité génétique est la même pour les 2 types de descendances : prépondérance des effets de dominance pour le rendement et à un moindre degré pour la longueur de la feuille; prépondérance des effets additifs pour tous les autres caractères. La variance d'additivité est de même importance en croisement intra- et interpopulation pour les caractères fortement héritables (mesures morphologiques et précocité), mais d'amplitude un peu plus variable pour le rendement. La corrélation entre les effets moyens des plantes mâles estimés en croisement intra- et interpopulation est positive pour tous les caractères, et d'autant plus faible que les caractères sont moins héritables. Les résultats sont en accord avec ceux de différentes expériences de sélection chez le maïs. Les conséquences de cette étude sur les méthodes de sélection sont discutées.
\end{abstract}

Zea mays L. - analyse hiérarchique - composantes de la variance - sélection récurrente réciproque

Summary - Intra- and interpopulation genetic variability in two forage maize populations. The intra- and interpopulation genetic variability was studied in 2 forage maize populations at $\mathrm{C}_{0}$ and $\mathrm{C}_{1}$ cycles of selection, from nested mating designs. The following traits were studied: flowering earliness, plant and ear heights, length and width of ear leaf, whole-plant yield and dry matter content. The nature of genetic variance was the same in intrapopulation and in interpopulation progenies: for yield and length of ear leaf, the dominance variance was significant; for the other traits, the additive variance explained most of the genetic variability. The intrapopulation and interpopulation additive variances were similar for highly heritable traits (heights and earliness) and were quite different for yield. The male plant average effects, estimated in intrapopulation and in interpopulation, were related: correlations were positive for all traits, lower for low heritable traits. Results were consistent with those reviewed from different selection experiments. Conclusions for breeding methods were then suggested.

Zea mays $L$. - nested design - variance components - reciprocal recurrent selection

\section{INTRODUCTION}

Sur le maïs (Zea mays L.), beaucoup d'études ont permis d'estimer et de décomposer la variabilité génétique. La population de référence est soit la population croisée avec elle-même (croisement intrapopulation), soit la population croisée avec une autre population (croisement interpopulation) (Hallauer \& Miranda, 1981). Mais peu de travaux ont comparé pour un même matériel la variabilité génétique révélée en croisements intra- et interpopulation. Une telle comparaison peut révéler l'existence d'une interaction entre les génotypes d'une population et la population testeur. Brun (1982) a défini les paramètres de cette interaction en la considérant comme un cas particulier d'interaction génotype $x$ milieu. Ces paramètres sont, d'une part, la corrélation entre

\footnotetext{
* Adresse actuelle : INRA, station de génétique et amélioration des plantes de Montpellier, domaine de Melgueil, 34130 Mauguio, France.
} 
les effets additifs estimés respectivement dans les descendances intrapopulation et interpopulation, et, d'autre part, l'importance relative des variances de ces effets additifs. De tels paramètres permettent de discuter du choix d'une méthode de sélection pour améliorer la valeur en croisement d'une population.

La présente étude a été réalisée sur 2 populations de maïs fourrage soumises à sélection. L'expérience a été entreprise pour analyser la variabilité génétique en croisement intra- et interpopulation. Son but était d'étudier l'efficacité de la sélection récurrente réciproque pour l'amélioration de la valeur propre des populations, et inversement l'efficacité d'une sélection intrapopulation pour l'amélioration de la valeur en combinaison des populations. Ainsi, le problème du choix du testeur pour améliorer les populations en vue de la création de variétés hybrides est sous-jacent dans cette étude.

\section{MATÉRIEL ET MÉTHODES}

\section{Matériel}

Deux populations synthétiques (SL19 et SL20) ont été constituées à la station d'amélioration des plantes de Lusignan par l'intercroisement d'une trentaine de lignées appartenant aux groupes de combinaisons "corné" et "denté", respectivement, dans un objectif d'amélioration de la valeur fourragère (Gallais et al., 1983). Ces 2 populations de maïs ont été sélectionnées en 1981, compte tenu des performances en croisement avec un testeur appartenant au groupe de combinaison complémentaire et sur la base d'un index combinant le rendement en matière sèche de la plante entière et la teneur en matière sèche. Le Tableau I donne les caractéristiques de rendement et de précocité pour ces 2 populations.

La variabilité génétique des populations a été étudiée au départ du programme de sélection (cycle $\mathrm{C}_{0}$ ) et après un cycle de sélection $\left(C_{1}\right)$. A chaque cycle, un plan de croisement hiérarchique a été effectué : une plante prise comme mâle a été croisée à 2 plantes de la même population et à 2 plantes de la population complémentaire. Les effectifs des plantes utilisées pour les différents plans de croisements figurent au Tableau II.

\section{Dispositif expérimental}

Les essais ont été réalisés au Moulon $(30 \mathrm{~km}$ au sud de Paris), en 1983 pour le cycle $C_{0}$ et en 1986 pour le cycle $C_{1}$, à la densité de 95000 plantes/ha. Les parcelles, constituées d'une seule ligne de $5 \mathrm{~m}$ de long, sont espacées de $0,8 \mathrm{~m}$. Les dispositifs expérimentaux sont des blocs incomplets à 2 répétitions en 1983 et 3 répétitions en 1986. Les descendances intra- et interpopulation d'une population ont été réparties dans les blocs, aléatoirement en 1983 et regroupées par plante mâle en 1986.

Tableau 1. Valeurs moyennes des 2 populations, de leur intercroisement au cycle $\mathrm{C}_{1}$ et de 2 hybrides commerciaux.

\begin{tabular}{|c|c|c|c|}
\hline $\begin{array}{l}\text { Populations } \\
\text { ou hybrides }\end{array}$ & $\begin{array}{c}\text { rendement } \\
\sec (t / h a)\end{array}$ & $\begin{array}{c}\text { teneur en } \\
\text { matière sèche (\%) }\end{array}$ & $\begin{array}{l}\text { date de } \\
\text { floraison (a) }\end{array}$ \\
\hline Dea & 17,32 & 28,18 & 27,87 \\
\hline intra cornée & 14,51 & 27,01 & 27,40 \\
\hline interpopulation & 15,29 & 27,20 & 27,94 \\
\hline intra dentée & 13,94 & 26,16 & 29,51 \\
\hline Brulouis & 14,76 & 30,40 & 23,80 \\
\hline
\end{tabular}

(a) : exprimée en nombre de jours à partir du 1 er juillet.

Tableau II. Nombre de plantes mâles étudiées par type de descendance.

\begin{tabular}{llccc}
\hline & & & Descendances \\
\cline { 3 - 5 } Population & Cycle & intrapopulation & interpopulation & intra- et interpopulation (a) \\
\hline \multirow{3}{*}{ cornée } & $\mathrm{C}_{0}$ & 37 & 31 & 23 \\
dentée & $\mathrm{C}_{0}$ & 34 & 36 & 24 \\
cornée & $\mathrm{C}_{1}$ & 42 & 42 & 42 \\
dentée & $\mathrm{C}_{1}$ & 36 & 36 & 36
\end{tabular}

(a) : issue de la même plante mâle. 


\section{Caractères observés}

Divers caractères permettant d'apprécier la valeur fourragère ont été mesurés. La précocité de floraison femelle (FLO) a été appréciée par la date à laquelle $50 \%$ des soies sont sorties (notée en nombres de jours à partir du 1er juillet). Différents caractères morphologiques ont été estimés à partir de la performance moyenne de 5 plantes par parcelle en 1983 et 8 plantes en 1986 : la hauteur totale (HT), mesurée du sol à la base de la fre ramification de la panicule, la hauteur d'insertion de l'épi supérieur (HE), la longueur (LOF) et la largeur (LAF) de la feuille de l'épi supérieur. Enfin, plusieurs caractères ont été observés à la récolte : la matière verte totale d'une parcelle a été pesée et le pourcentage de matière sèche (MS) a été estimé par séchage à l'étuve d'un échantillon de $800 \mathrm{~g}$ de matière verte. Pour l'essai de 1983, le rendement frais (RF) a été corrigé pour tenir compte de l'irrégularité du peuplement. La correction a été faite selon le principe de l'analyse de covariance :

$$
\mathrm{RF} \text { corrigé }=\mathrm{RF}-\beta(\mathrm{NP}-\overline{\mathrm{NP}})
$$

où $\beta$ est le coefficient de régression entre le rendement et le peuplement estimé sur les données brutes, NP est le nombre de pieds par parcelle, et $\overline{\mathrm{NP}}$ est la moyenne du nombre de pieds par parcelle de l'essai.

Le rendement sec (RS) résulte du produit du rendement frais $(\mathrm{RF})$ et de la teneur en matière sèche.

\section{Interprétation}

Sur chaque type de descendance, des analyses de variance multivariables ont été effectuées en modèle mixte en utilisant la méthode d'Henderson III. Les facteurs génétiques ont été considérés comme aléatoires et les facteurs dus au terrain comme fixes. Ces analyses ont conduit aux estimations des variances entre plantes mâles $\left(\widehat{\sigma}^{2} \mathrm{M}\right)$ et entre plantes femelles hiérarchisées aux plantes mâles ( $\left.\widehat{\sigma}^{2} \mathrm{~F} / \mathrm{M}\right)$.

L'introduction d'un modèle génétique simple faisant l'hypothèse d'absence d'épistasie et d'effets réciproques permet de décomposer la variabilité en termes de variances d'additivité et de dominance (modèle de Kempthorne (1957) pour les croisements intrapopulation; modèle de Griffing (1962) pour les croisements interpopulation). L'hypothèse d'absence d'épistasie est justifiée, compte tenu des résultats de la bibliographie : différents auteurs ont noté des effets d'épistasie, mais ceux-ci sont relativement faibles par rapport à ceux de dominance et d'additivité (Darrah \& Hallauer, 1972; Moreno-Gonzalez \& Dudley, 1981; Hébert, 1986; Melchinger et al., 1986). Même en présence d'épistasie, le modèle, intégrant seulement des effets d'additivité et de dominance, peut conserver une valeur prédictive (Gallais, 1978a). L'hypothèse d'absence d'effets réciproques est elle aussi justifiée pour les caractères étudiés (Melchinger et al., 1985; Hébert, 1986).

Les variances d'additivité $\left(\widehat{\sigma}^{2} \mathrm{~A}\right)$ et de dominance

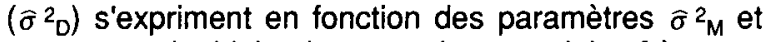
$\widehat{\sigma}^{2} F M$, par le biais des covariances pleins-frères et demi-frères. Dans les croisements intrapopulation, les expressions pour la population cornée, par exemple, sont les suivantes:

$\widehat{\sigma}_{M}^{2}=4 c \widehat{\sigma}_{M}^{2}$ et $\widehat{\sigma}_{D}^{2}=4\left({ }_{c c} \widehat{\sigma}_{F M}^{2}-{ }_{c c} \widehat{\sigma}_{M}^{2}\right)$
Dans les croisements interpopulation, les résultats des deux populations sont pris en compte simultanément :

$$
\begin{aligned}
& \widehat{\sigma}_{M}^{2}=2\left(c_{c d} \widehat{\sigma}_{M}^{2}+d c \widehat{\sigma}_{M}^{2}\right) \\
& \text { et } \widehat{\sigma}_{D}^{2}=2\left({ }_{c d} \widehat{\sigma}{ }_{F M}^{2}+{ }_{d c} \widehat{\sigma} \stackrel{F M M}{2}_{d c}^{2} \widehat{\sigma}_{M}^{2}-c_{c d} \widehat{\sigma}_{M}^{2}\right)
\end{aligned}
$$

Dans le cas de croisements interpopulation, les expressions des covariances pleins-frères et demifrères ont été données par Gallais (1978b).

Dans les expressions précédentes, les indices placés devant les composantes de la variance indiquent le type de descendance considéré : " $c c$ " pour la descendance de la population cornée croisée en intrapopulation, "cd" pour la descendance de la population cornée (mâle) croisée avec la population dentée (femelle), "dc" pour la descendance de la population dentée (mâle) croisée avec la population cornée (femelle).

\section{RÉSULTATS ET DISCUSSION}

\section{Décomposition de la variance génétique}

\section{Approche de l'estimation des composantes de la variance génétique}

A partir des estimations des composantes mâle et femelle de la variance données au Tableau III, l'importance relative de l'additivité et de la dominance a été discutée pour l'ensemble des caractères au Tableau IV.

Pour le rendement sec mesuré sur la descendance intrapopulation dentée au cycle $\mathrm{C}_{0}$, les effets génétiques mâle et femelle ne sont pas significatifs à $5 \%$. Dans ce cas (noté «-» dans le Tableau IV), la variabilité génétique n'a pas été analysée.

Dans les cas où la variance entre mâles $\left(\widehat{\sigma}^{2} \mathrm{M}\right)$ est proche de 0 et la variance entre femelles $\left(\widehat{\sigma}^{2} F M\right)$ est différente de 0 , la variance d'additivité a été considérée comme négligeable par rapport à la variance de dominance. Ces cas (notés "D" dans le Tableau IV) se présentent fréquemment pour le rendement sec et la longueur de la feuille.

Pour tous les autres caractères, les estimations de la variance de dominance sont négatives. Ces valeurs peuvent être expliquées par une imprécision des estimations et (ou) par une sous-estimation de la variance entre femelles.

Pour tous les caractères, l'imprécision des estimations du fait du plan de croisement a pu conduire à des estimations négatives de la variance de dominance. Le plan hiérarchique fournit des estimations moins précises de la variance de dominance que le plan factoriel 
Tableau III. Estimation des composantes mâle $\left(\bar{\sigma}^{2} \mathrm{M}\right)$ et femelle $\left(\bar{\sigma}^{2} \mathrm{FM}\right)$ de la variance avec leurs écarts types (entre parenthèses) pour les cycles $C_{0}$ et $C_{1}$.

\begin{tabular}{|c|c|c|c|c|c|c|c|c|}
\hline & \multicolumn{4}{|c|}{$\hat{\sigma}^{2} M$} & \multicolumn{4}{|c|}{$\bar{\sigma}^{2} \mathrm{~F} / \mathrm{M}$} \\
\hline & $\begin{array}{c}\text { intra } \\
\text { cornée }\end{array}$ & $\begin{array}{c}\text { inter } \\
\text { cornée }\end{array}$ & $\begin{array}{c}\text { inter } \\
\text { dentée }\end{array}$ & $\begin{array}{c}\text { intra } \\
\text { dentée }\end{array}$ & $\begin{array}{c}\text { intra } \\
\text { cornée }\end{array}$ & $\begin{array}{l}\text { inter } \\
\text { cornée }\end{array}$ & $\begin{array}{c}\text { inter } \\
\text { dentée }\end{array}$ & $\begin{array}{c}\text { intra } \\
\text { dentée }\end{array}$ \\
\hline \multicolumn{9}{|c|}{ essai de 1983 : cycle $C_{0}$} \\
\hline MS & $\begin{array}{l}2,78^{*} \\
(1,02)\end{array}$ & $\begin{array}{l}2,47^{*} \\
(1,02)\end{array}$ & $\begin{array}{l}7,06^{*} \\
(3,02)\end{array}$ & $\begin{array}{c}11,45^{*} \\
(3,75)\end{array}$ & $\begin{array}{l}1,03 \text { * } \\
(0,67)\end{array}$ & $\begin{array}{l}0,86 \\
(0,69)\end{array}$ & $\begin{array}{r}1,90 \\
(2,48)\end{array}$ & $\begin{array}{r}-2,45 \\
(2,24)\end{array}$ \\
\hline RS & $\begin{array}{l}1,41^{\star} \\
(0,78)\end{array}$ & $\begin{array}{r}1,06 \\
(0,55)\end{array}$ & $\begin{array}{l}0,37 \\
(0,93)\end{array}$ & $\begin{array}{r}-0,01 \\
(0,65)\end{array}$ & $\begin{array}{r}1,12 \\
(0,76)\end{array}$ & $\begin{array}{r}-0,16 \\
(0,57)\end{array}$ & $\begin{array}{l}2,12^{*} \\
(1,31)\end{array}$ & $\begin{array}{r}1,33 \\
(1,01)\end{array}$ \\
\hline RF & $\begin{array}{l}53,47^{*} \\
(20,48)\end{array}$ & $\begin{array}{l}25,95 \text { * } \\
(10,83)\end{array}$ & $\begin{array}{l}52,93 \text { * } \\
(20,82)\end{array}$ & $\begin{array}{l}56,11^{*} \\
(17,72)\end{array}$ & $\begin{array}{l}33,61 \text { * } \\
(13,40)\end{array}$ & $\begin{array}{r}7,53 \\
(7,83)\end{array}$ & $\begin{array}{l}46,01 \\
(13,49)\end{array}$ & $\begin{array}{c}14,58 \text { * } \\
(6,86)\end{array}$ \\
\hline HT & $\begin{array}{l}52,56^{*} \\
(26,22)\end{array}$ & $\begin{array}{l}61,65^{\star} \\
(28,91)\end{array}$ & $\begin{array}{l}98,00 \text { * } \\
(38,48)\end{array}$ & $\begin{array}{c}172,65 \text { * } \\
(54,95)\end{array}$ & $\begin{array}{l}24,72 \\
(24,86)\end{array}$ & $\begin{array}{l}27,45 \\
(23,16)\end{array}$ & $\begin{array}{l}49,82 \text { * } \\
(26,33)\end{array}$ & $\begin{array}{l}37,25 \text { * } \\
(22,48)\end{array}$ \\
\hline $\mathrm{HE}$ & $\begin{array}{l}48,31^{\star} \\
(18,63)\end{array}$ & $\begin{array}{l}16,08 \\
(15,00)\end{array}$ & $\begin{array}{l}66,84 \text { * } \\
(25,88)\end{array}$ & $\begin{array}{c}105,73 \text { * } \\
(34,57)\end{array}$ & $\begin{array}{r}12,49 \\
(13,56)\end{array}$ & $\begin{array}{l}31,86^{*} \\
(17,23)\end{array}$ & $\begin{array}{l}35,84 \text { * } \\
(17,18)\end{array}$ & $\begin{array}{l}17,83 \\
(16,01)\end{array}$ \\
\hline LOF & $\begin{array}{l}4,17 \\
(3,09)\end{array}$ & $\begin{array}{r}1,46 \\
(2,61)\end{array}$ & $\begin{array}{l}8,62 \\
(6,10)\end{array}$ & $\begin{array}{c}23,99 \text { * } \\
(7,89)\end{array}$ & $\begin{array}{l}7,14 \text { * } \\
(3,39)\end{array}$ & $\begin{array}{l}5,67^{*} \\
(3,48)\end{array}$ & $\begin{array}{c}20,31 \text { * } \\
(6,25)\end{array}$ & $\begin{array}{l}7,13 \text { * } \\
(3,49)\end{array}$ \\
\hline LAF & $\begin{array}{r}7,46 \\
(9,50)\end{array}$ & $\begin{array}{c}17,49 \text { * } \\
(7,60)\end{array}$ & $\begin{array}{c}15,20 \text { * } \\
(8,11)\end{array}$ & $\begin{array}{l}70,35 \text { * } \\
(25,80)\end{array}$ & $\begin{array}{l}31,64 \text { * } \\
(11,67)\end{array}$ & $\begin{array}{r}1,81 \\
(6,00)\end{array}$ & $\begin{array}{c}15,11 \text { * } \\
(7,46)\end{array}$ & $\begin{array}{r}2,42 \\
(17,18)\end{array}$ \\
\hline FLO & $\begin{array}{l}2,94^{\star} \\
(1,17)\end{array}$ & $\begin{array}{c}2,27^{*} \\
(1,17)\end{array}$ & $\begin{array}{c}10,90^{*} \\
(3,14)\end{array}$ & $\begin{array}{l}8,39^{*} \\
(2,67)\end{array}$ & $\begin{array}{l}1,67^{*} \\
(0,83)\end{array}$ & $\begin{array}{l}2,80 \text { * } \\
(0,94)\end{array}$ & $\begin{array}{r}1,28 \\
(1,00)\end{array}$ & $\begin{array}{l}1,86 \text { * } \\
(1,09)\end{array}$ \\
\hline essai & $6:$ cycle $C_{1}$ & & & & & & & \\
\hline MS & $\begin{array}{c}1,02 \text { * } \\
(0,33)\end{array}$ & $\begin{array}{c}0,72 \\
(0,28)\end{array}$ & $\begin{array}{l}3,41 \text { * } \\
(1,00)\end{array}$ & $\begin{array}{l}3,46 \text { * } \\
(1,16)\end{array}$ & $\begin{array}{l}0,24^{*} \\
(0,17)\end{array}$ & $\begin{array}{l}0,15 \\
(0,15)\end{array}$ & $\begin{array}{c}0,53^{*} \\
(0,34)\end{array}$ & $\begin{array}{l}1,48 \text { * } \\
(0,49)\end{array}$ \\
\hline RS & $\begin{array}{r}-0,05 \\
(0,35)\end{array}$ & $\begin{array}{r}-0,20 \\
(0,34)\end{array}$ & $\begin{array}{r}0,30 \\
(0,40)\end{array}$ & $\begin{array}{c}0,03 \\
(0,31)\end{array}$ & $\begin{array}{l}1,29 \text { * } \\
(0,51)\end{array}$ & $\begin{array}{l}0,99 \text { * } \\
(0,47)\end{array}$ & $\begin{array}{c}0,84 \\
(0,44)\end{array}$ & $\begin{array}{c}1,00^{\star} \\
(0,42)\end{array}$ \\
\hline RF & $\begin{array}{r}6,50 \\
(7,96)\end{array}$ & $\begin{array}{r}9,60 \\
(7,87)\end{array}$ & $\begin{array}{c}24,73 \text { * } \\
(9,69)\end{array}$ & $\begin{array}{l}23,06 \\
(10,17)\end{array}$ & $\begin{array}{l}25,82^{*} \\
(10,06)\end{array}$ & $\begin{array}{c}18,61 \\
(8,47)\end{array}$ & $\begin{array}{c}13,52 \text { * } \\
(5,64)\end{array}$ & $\begin{array}{c}21,31 \text { * } \\
(7,62)\end{array}$ \\
\hline HT & $\begin{array}{l}38,04 \text { * } \\
(16,41)\end{array}$ & $\begin{array}{l}57,41 \text { * } \\
(20,00)\end{array}$ & $\begin{array}{l}64,27^{*} \\
(24,42)\end{array}$ & $\begin{array}{l}39,48 \text { * } \\
(18,84)\end{array}$ & $\begin{array}{c}28,24^{\star} \\
(9,68)\end{array}$ & $\begin{array}{c}17,53^{*} \\
(8,07)\end{array}$ & $\begin{array}{c}27,04 \\
(9,79)\end{array}$ & $\begin{array}{l}38,20 \text { * } \\
(11,70)\end{array}$ \\
\hline HE & $\begin{array}{l}28,61 \text { * } \\
(13,20)\end{array}$ & $\begin{array}{l}47,99 \text { * } \\
(18,25)\end{array}$ & $\begin{array}{l}53,38 \text { * } \\
(21,31)\end{array}$ & $\begin{array}{l}80,04 \text { * } \\
(24,11)\end{array}$ & $\begin{array}{c}27,87 \text { * } \\
(9,76)\end{array}$ & $\begin{array}{c}25,40^{*} \\
(9,22)\end{array}$ & $\begin{array}{l}41,53^{*} \\
(13,28)\end{array}$ & $\begin{array}{c}21,27 \text { * } \\
(6,63)\end{array}$ \\
\hline LOF & $\begin{array}{l}4,20^{\star} \\
(2,19)\end{array}$ & $\begin{array}{l}8,20^{*} \\
(2,99)\end{array}$ & $\begin{array}{l}3,90 \\
(2,69)\end{array}$ & $\begin{array}{r}4,96 \\
(3,37)\end{array}$ & $\begin{array}{l}3,64 \text { * } \\
(1,64)\end{array}$ & $\begin{array}{l}4,64 \text { * } \\
(1,73)\end{array}$ & $\begin{array}{l}8,78^{*} \\
(2,44)\end{array}$ & $\begin{array}{c}12,09 \text { * } \\
(3,33)\end{array}$ \\
\hline LAF & $\begin{array}{c}17,80 \text { * } \\
(7,23)\end{array}$ & $\begin{array}{c}17,80^{*} \\
(6,93)\end{array}$ & $\begin{array}{c}30,84 \text { * } \\
(9,46)\end{array}$ & $\begin{array}{c}18,53^{*} \\
(5,80)\end{array}$ & $\begin{array}{c}14,31 \\
(5,00)\end{array}$ & $\begin{array}{c}13,55 \\
(4,56)\end{array}$ & $\begin{array}{c}8,03^{*} \\
(3,21)\end{array}$ & $\begin{array}{l}4,29 \text { * } \\
(2,50)\end{array}$ \\
\hline FLO & $\begin{array}{l}1,48^{*} \\
(0,67)\end{array}$ & $\begin{array}{l}2,94 \text { * } \\
(1,05)\end{array}$ & $\begin{array}{l}6,35 \text { * } \\
(2,02)\end{array}$ & $\begin{array}{l}6,822^{*} \\
(2,07)\end{array}$ & $\begin{array}{r}0,59 \\
(0,51)\end{array}$ & $\begin{array}{l}1,29 \text { * } \\
(0,48)\end{array}$ & $\begin{array}{l}2,03 \text { * } \\
(0,74)\end{array}$ & $\begin{array}{c}1,84 \text { * } \\
(0,60)\end{array}$ \\
\hline
\end{tabular}

\footnotetext{
* : significatif à $5 \%$,
}

MS : teneur en matière sèche; RS : rendement sec; RF : rendement frais; HT : hauteur totale; HE : hauteur de l'épi; LOF : longueur de la feuille de l'épi; LAF : largeur de la feuille de l'épi; FLO : date de floraison;. 
Tableau IV. Interprétation qualitative des estimations des composantes de la variance génétique.

\begin{tabular}{|c|c|c|c|c|c|c|c|c|}
\hline descendances & $M S$ & RS & $R F$ & $H T$ & $H E$ & $L O F$ & $L A F$ & $F L O$ \\
\hline \multicolumn{9}{|l|}{ cycle $C_{0}$} \\
\hline intra «corné» & $A$ & $x$ & $A$ & $x$ & $x$ & $\mathrm{D}$ & $D$ & $A$ \\
\hline inter & $x$ & $\mathrm{D}$ & $A$ & A & A & $\mathrm{D}$ & A & A \\
\hline intra «denté" & $x$ & - & A & A & $x$ & A & $x$ & A \\
\hline \multicolumn{9}{|l|}{ cycle $C_{1}$} \\
\hline intra «corné» & $A$ & $D$ & $D$ & $A$ & $A$ & $A$ & A & $x$ \\
\hline inter & $A$ & $D$ & A & $A$ & A & $A$ & A & $A$ \\
\hline intra “denté» & $A$ & $D$ & $A$ & A & A & $\mathrm{D}$ & A & A \\
\hline
\end{tabular}

$-: \sigma^{2} F / M=0$ et $\sigma_{M}^{2} \approx 0:$ variabilité génétique non significative; $x: \sigma^{2} F / M \approx 0$ et $\sigma^{2} M \neq 0:$ voir texte; $D:$ caractère à variabilité surtout dominante; $A$ : caractère à variabilité essentiellement additive; l'explicitation des abréviations des caractères est sous le Tableau II!

(Bridges \& Knapp, 1987). Notons que ce plan est le seul possible dans le cas de plantes $S_{0}$ non prolifiques. De plus, le choix de seulement 2 plantes femelles pour une même plante mâle a dû accentuer cette imprécision. Ce facteur contribue à surestimer ou à sous-estimer les valeurs des composantes de la variance. Dans cette expérience, la variance des effets de dominance est très souvent estimée négativement. D'autres facteurs ont dû intervenir. Les cas où $\widehat{\sigma}^{2} \mathrm{~F} M \mathrm{M} \approx 0$ et $\widehat{\sigma}^{2} M \neq 0\left(\widehat{\sigma}_{A}{ }^{2}+\widehat{\sigma}_{D}^{2} \approx 0\right.$ et $\left.\widehat{\sigma}_{A}^{2} \neq 0\right)$ sont difficilement explicables avec le maintien de toutes les hypothèses des modèles génétique et statistique. Les facteurs susceptibles de conduire à une sous-estimation de la variance de dominance sont l'échantillonnage non aléatoire des plantes (Lindsey et al., 1962), l'existence d'effets réciproques et de problèmes expérimentaux. Parmi les caractères où une telle situation se présente (notés "X" dans le Tableau IV), on notera la teneur en matière sèche au cycle $C_{0}$ et la date de floraison au cycle $C_{1}$. L'hypothèse d'homogamie semble possible : les 2 plantes femelles croisées à une même plante mâle auraient été choisies dans une même gamme de précocité, bien que les 2 dates de semis en pépinière soient différentes. De ce fait, la variance entre femelles est réduite et conjointement la variance entre mâles est surestimée, ceci pour le caractère considéré et corrélativement pour les autres caractères du fait de leur liaison avec la précocité.

Ces différents facteurs ont dû contribuer à l'obtention d'estimations négatives de la variance de dominance. Ainsi, la décomposition de la variabilité génétique en termes d'additivité et de dominance est délicate et seules des conclusions qualitatives ont été données. Dans les cas où $\widehat{\sigma}^{2} F / M \neq 0$ et $\widehat{\sigma}^{2} M \neq 0$ (notés «A» dans le Tableau IV), la variabilité a été jugée comme étant essen- tiellement additive. Ces cas sont observés pour la hauteur et la date de floraison.

\section{Importance relative de l'additivité et de la dominance dans la variabilité génétique}

\section{Variations selon le cycle de sélection}

Malgré les problèmes de précision, la part relative de l'additivité et de la dominance dans la variabilité génétique apparaît assez stable d'un cycle à l'autre (Tableau IV). Une modification de la nature de la variabilité est observée pour le rendement frais dans la descendance «intra» de la population cornée et pour la longueur de la feuille dans la descendance «intra" de la population dentée dans le sens suivant : la variance d'additivité est importante au cycle $\mathrm{C}_{0}$, alors que des effets de dominance sont détectés au cycle $C_{1}$. Bien qu'un seul cycle de sélection ait été réalisé, ces résultats sont conformes à ce qui est attendu sous l'effet de plusieurs cycles de sélection : augmentation des effets de dominance et diminution des effets d'additivité. De tels résultats ont été observés après plusieurs cycles de sélection chez le maïs (Singh et al., 1985; Reeder et al., 1987).

Par ailleurs, dans la population cornée, pour la longueur de la feuille (croisement interpopulation) et pour la largeur de la feuille (croisement intrapopulation), la variabilité génétique est expliquée essentiellement par des effets d'additivité au cycle $C_{1}$, alors que des effets de dominance ont été notés au cycle $C_{0}$. Ces résultats, qui sont plus difficiles à expliquer, pourraient correspondre à des variations aléatoires; celles-ci pourraient être expliquées par le fait que les effets du cycle et de l'année expérimentale sont confondus. 


\section{Variations selon le caractère}

Les caractères pour lesquels la variance de dominance est non négligeable sont le rendement sec et la longueur de la feuille. Le rendement (en grain) est reconnu comme étant déterminé à la fois par des effets d'additivité et de dominance (Hallauer \& Miranda, 1981); la longueur de la feuille, beaucoup moins étudiée, est citée comıme étant à déterminisme plutôt additif. Globalement, les résultats apparaissent assez en accord avec ceux de la bibliographie : prépondérance de la variance d'additivité pour la plupart des caractères hormis le rendement.

\section{Variations selon le type de descendance}

Pour les caractères où la variance de dominance est significative, la variabilité génétique n'est pas systématiquement expliquée par les mêmes effets en croisement intrapopulation et en croisement interpopulation : pour la longueur de la feuille au cycle $\mathrm{C}_{0}$, la variance de dominance est significative seulement en croisement interpopulation; par contre pour le rendement frais (cycle $\mathrm{C}_{1}$ ), la longueur de la feuille (cycle $\mathrm{C}_{1}$ ) et la largeur de la feuille (cycle $\mathrm{C}_{0}$ ), la variance de dominance n'est significative qu'en croisement intrapopulation. Du fait de la nature essentiellement additive de la variabilité génétique pour la majorité des caractères, les cas d'expression différente de la variabilité selon le type de descendance sont assez rares.
Dans les quelques expériences publiées pour lesquelles le rapport $\left(\widehat{\sigma}_{D}^{2} / \widehat{\sigma}_{A}{ }_{A}\right)$ a pu être calculé (Tableau V), la hauteur de l'épi et la précocité de floraison présentent une variabilité essentiellement additive en croisement intrapopulation comme en croisement interpopulation. Dans la plupart des populations citées au Tableau V, le rendement en grain montre une variance de dominance inférieure à la variance d'additivité dans les 2 types de descendance, sauf pour les populations Golden Jewel et Osterland's Yellow Dent. Dans un seul cas, la population J607, l'importance relative des variances des effets génétiques diffère selon le type de descendance.

Dans ces expériences comme dans cette étude, il est difficile de conclure à une expression différente de la variabilité génétique selon le type de descendance.

\section{Importance relative des variances d'additivi- té en croisement intra- et interpopulation}

\section{Résultats expérimentaux}

Pour comparer 2 composantes de la variance, il est possible de comparer les bornes de leur intervalle de confiance. Celles-ci ont été données par Scheffé (1959) dans le cas d'un dispositif équilibré. Du fait de l'étendue des intervalles de confiance, seules des différences très importantes entre les 2 variances seront révélées.

Tableau V. Rapport de la variance de dominance sur la variance d'additivité en croisement intra- et interpopulation

\begin{tabular}{|c|c|c|c|c|c|c|c|}
\hline \multirow[t]{2}{*}{ Référencesa } & \multirow[t]{2}{*}{ Population } & \multicolumn{2}{|c|}{$\begin{array}{l}\text { Rendement } \\
\text { grain }\end{array}$} & \multicolumn{2}{|c|}{$\begin{array}{l}\text { Hauteur } \\
\text { de l'épi }\end{array}$} & \multicolumn{2}{|c|}{$\begin{array}{l}\text { Date de } \\
\text { floraison }\end{array}$} \\
\hline & & intra & inter & intra & inter & intra & inter \\
\hline Robinson et al. (1958) & $\begin{array}{l}\text { Jarvis } \\
\text { Indian Chief }\end{array}$ & $\begin{array}{l}0,2 \\
0,5\end{array}$ & $\begin{array}{l}0,8 \\
0,6\end{array}$ & & & & \\
\hline Compton et al. (1965) & $\begin{array}{l}\text { Golden Republic } \\
\text { Barber Reid }\end{array}$ & $\begin{array}{l}0,4 \\
0,7\end{array}$ & $\begin{array}{l}0,3 \\
0,3\end{array}$ & $\begin{array}{l}0,6 \\
0\end{array}$ & $\begin{array}{l}0 \\
0\end{array}$ & $\begin{array}{l}0,4 \\
0,1\end{array}$ & $\begin{array}{l}0,1 \\
0,1\end{array}$ \\
\hline Goodrich et al. (1975) & $\begin{array}{l}\text { Golden Jewel } \\
\text { Osterland's } \\
\text { Yellow Dent }\end{array}$ & $\begin{array}{l}2,0 \\
6,9\end{array}$ & $\begin{array}{l}4,9 \\
4,0\end{array}$ & $\begin{array}{l}0 \\
0\end{array}$ & $\begin{array}{l}0 \\
0\end{array}$ & $\begin{array}{l}0 \\
0\end{array}$ & $\begin{array}{l}0 \\
0\end{array}$ \\
\hline Singh et al. (1984) & $\begin{array}{l}\text { MAS } \\
\text { J607 }\end{array}$ & $\begin{array}{l}0,5 \\
5,8\end{array}$ & $\begin{array}{l}0,8 \\
0,7\end{array}$ & $\begin{array}{l}0 \\
0,6\end{array}$ & $\begin{array}{l}0,3 \\
0,3\end{array}$ & $\begin{array}{l}0 \\
0\end{array}$ & $\begin{array}{l}0,4 \\
0,5\end{array}$ \\
\hline Moreno-Gonzalez (1988) b & $\begin{array}{l}7 \text { lignées cornées } \\
7 \text { lignées dentées }\end{array}$ & $\begin{array}{l}0,2 \\
0,4\end{array}$ & $\begin{array}{l}0,3 \\
0,4\end{array}$ & & & $\begin{array}{l}0,6 \\
0,1\end{array}$ & $\begin{array}{l}0,1 \\
0,2\end{array}$ \\
\hline
\end{tabular}

1: caractère non mesuré; $\mathrm{a}:$ le rapport $\left(\tilde{\sigma}^{2} / \hat{\sigma}^{2} \mathrm{~A}\right)$ a été calculé à partir des données publiées; ${ }^{\mathrm{b}}$ : plans diallèle et factoriel. 
Dans cette expérience, les intervalles de confiance à $95 \%$ des variances entre plantes mâles en croisement intrapopulation et en croisement interpopulation se recoupent. Les variances d'additivité correspondantes sont donc du même ordre de grandeur. La seule tendance observée est une légère supériorité des variances d'additivité en croisement intrapopulation pour les caractères morphologiques dans la population dentée au cycle $\mathrm{C}_{0}$ (Tableau III).

Dans les autres expériences comparant les 2 types de descendance (Tableau VI), les rapports des variances d'additivités sont proches de 1 pour la hauteur et la précocité, mais s'écartent plus souvent de 1 pour le rendement. Si l'on considère de façon approximative l'étendue des intervalles de confiance, seuls des rapports supérieurs à 4 (ou inférieurs à 0,25 ) indiquent des différences nettes entre les 2 types de variance (une variance étant statistiquement comprise à $95 \%$ entre la moitié et le double de son estimation). Pour le rendement, les variances d'additivité estimées en croisement intra- et interpopulation apparaissent donc peu différentes entre elles et aucune tendance nette ne se dégage en faveur d'un type. Ce résultat avait déjà été mis en évidence pour le rendement en grain chez le maïs (Obilana et al., 1979); Miranda-Filho \& Paterniani, 1983).

\section{Interprétation}

La variance dépend de la fréquence des gènes, du degré de dominance et des effets géniques. Pour aider à l'interprétation des résultats, les variances d'additivité ont été exprimées dans le cadre d'un modèle génétique simple.

Pour un caractère déterminé par un locus biallèlique, les expressions des variances additives

Tableau VI. Rapport de la variance additive intrapopulation sur la variance additive interpopulation ( $r$ ) et hétérosis $(H)$ entre les 2 populations.

\begin{tabular}{|c|c|c|c|c|c|c|c|}
\hline \multirow[t]{2}{*}{ Références } & \multirow[t]{2}{*}{ Population } & \multicolumn{2}{|c|}{$\begin{array}{l}\text { Rendement } \\
\text { grain }\end{array}$} & \multicolumn{2}{|c|}{$\begin{array}{l}\text { Hauteur } \\
\text { de l'épi }\end{array}$} & \multicolumn{2}{|c|}{$\begin{array}{l}\text { Date de } \\
\text { floraison }\end{array}$} \\
\hline & & $r$ & $H$ & $r$ & $H$ & $r$ & $H$ \\
\hline \multirow[b]{2}{*}{ Robinson et al. (1958) } & Jarvis & 2,5 & \multirow[b]{2}{*}{$26,0 \%$} & 1 & & 1 & \\
\hline & Indian Chief & 1,5 & & 1 & & 1 & \\
\hline \multirow{2}{*}{ Compton et al. (1965) } & Golden Republic & 0,9 & \multirow{2}{*}{$6,9 \%$} & 0,6 & \multirow{2}{*}{$0,8 \%$} & 0,9 & \\
\hline & Barber Reid & 0,6 & & 1,2 & & 0,7 & $0,4 \%$ \\
\hline \multirow{2}{*}{ Goodrich et al. (1975) } & Golden Jewel & 4,2 & \multirow{2}{*}{$27,2 \%$} & 0,7 & \multirow{2}{*}{$4,9 \%$} & 1,1 & \multirow{2}{*}{$-1,1 \%$} \\
\hline & $\begin{array}{l}\text { Osterland's } \\
\text { Yellow Dent }\end{array}$ & 0,7 & & 1,0 & & 1,0 & \\
\hline \multirow{2}{*}{ Singh et al. (1984) } & MAS & 1,2 & \multirow{2}{*}{$15,4 \%$} & 0,9 & \multirow{2}{*}{$4,8 \%$} & 1,8 & \multirow{2}{*}{1} \\
\hline & $\mathrm{J} 607$ & 0,3 & & 0,8 & & 1,8 & \\
\hline \multirow{2}{*}{ Moreno-Gonzalez (1988)a } & ( 7 lignées cornées & 2,6 & \multirow{2}{*}{1} & 1 & & 0,5 & \multirow{2}{*}{1} \\
\hline & 7 lignées dentées & 0,7 & & 1 & & 1,9 & \\
\hline \multirow{4}{*}{ cette étude } & \{ Cornée & $1,3^{b}$ & \multirow[t]{2}{*}{$9,3 \%$} & 3,0 & \multirow[t]{2}{*}{$2,5 \%$} & 1,3 & \multirow[t]{2}{*}{$0,2 \%$} \\
\hline & Dentée & - & & 1,6 & & 0,8 & \\
\hline & Cornée & - & \multirow{2}{*}{$7,5 \%$} & 0,6 & \multirow{2}{*}{$2,4 \%$} & 0,5 & \multirow{2}{*}{$-1,8 \%$} \\
\hline & Dentée & - & & 1,5 & & 1,1 & \\
\hline
\end{tabular}

I: caractère non mesuré; $a$ : plans factoriel et diallèle; $b$ : rendement fourrage; $-: \hat{\sigma}^{2} M=0$ 
en croisement intrapopulation $\left(\sigma^{2}{ }_{A 11}\right)$ et en croisement interpopulation $\left(\sigma^{2} \mathrm{~A} 12\right)$ sont :

$$
\begin{aligned}
& \sigma_{\mathrm{A} 11}^{2}=2 p_{1} q_{1}\left[a-\left(p_{1}-q_{1}\right) d\right]^{2} \text { et } \\
& \sigma_{\mathrm{A} 12}^{2}=2 p_{1} q_{1}\left[a-\left(p_{2}-q_{2}\right) d\right]^{2}
\end{aligned}
$$

où $p_{1}$ (resp. $p_{2}$ ) est la fréquence de l'allèle favorable dans la population 1 (resp. 2) et $q_{1}$ (resp. $q_{2}$ ) est la fréquence de l'allèle défavorable, $a$ est la moitié de la différence entre les performances des 2 homozygotes, et $d$ est la performance de l'hétérozygote. La différence entre ces deux expressions est :

$$
\begin{array}{r}
\sigma_{\mathrm{A} 11}^{2}-\sigma_{\mathrm{A} 12}^{2}=8 p_{1} q_{1}\left(p_{2}-p_{1}\right) \\
{\left[a-\left(p_{1}+p_{2}-1\right) d\right] d}
\end{array}
$$

Dans le cas de dominance favorable $(d>0)$ et d'absence de superdominance $(d<a)$, la variance d'additivité en croisement intrapopulation est supérieure à la variance d'additivité en croisement interpopulation si la fréquence de l'allèle favorable est plus faible dans la population étudiée que dans la population partenaire. Des variances très différentes apparaîtront pour de fortes différences de fréquence de l'allèle favorable et un degré de dominance élevé, c'est-àdire lorsque l'hétérosis $(H)$ entre les 2 populations est important pour le caractère considéré $\left(\mathrm{H}=\left(p_{1}-p_{2}\right)^{2} \mathrm{~d}\right.$; Falconer, 1960).

Dans ce modèle simpliste, il existe une relation entre le niveau d'hétérosis du caractère et l'amplitude relative des variances $\sigma^{2}{ }_{\mathrm{A} 11}$ et $\sigma^{2} \mathrm{~A} 12$, ces paramètres correspondant à 2 manifestations de linteraction existant entre les populations.

Dans notre expérience, le rapport des variances d'additivité (estimé par $\widehat{\sigma}_{M}^{2}$ intra / $\widehat{\sigma}_{M}^{2}$ inter) est proche de 1 pour la majorité des caractères, dont le rendement (Tableau III). Par contre, dans la bibliographie (Tableau VI), il ressort que le rapport des 2 variances est le plus grand pour le rendement, caractère qui est le plus hétérotique.

\section{Corrélations phénotypiques entre les effets moyens estimés en croisement intra- et interpopulation}

Le Tableau VII donne les estimations des coefficients de corrélation phénotypiques entre les effets moyens des plantes mâles estimées en croisement intra- et interpopulation. Du fait de l'imprécision des estimations, les valeurs obtenues pour le coefficient de corrélation génétique sont souvent supérieures à 1 . Aussi, seules les valeurs phénotypiques ont été analysées. Toutes les corrélations phénotypiques sont positives (sauf pour la largeur de la feuille dans la population dentée au cycle $\mathrm{C}_{0}$ ) et la plupart sont significatives à $5 \%$. Sur les 2 cycles de sélection, les estimations du coefficient de corrélation sont similaires. La sélection réalisée entre $C_{0}$ et $C_{1}$ n'a pas fait évoluer ce paramètre; ce résultat était prévisible après si peu de cycles de sélection récurrente réciproque. Le coefficient de corrélation est relativement plus élevé pour les caractères plus héritables, tels que les mesures morphologiques ou la précocité, que pour le rendement, à faible héritabilité. Mais l'imprécision de l'estimation du rendement peut conduire à des estimations faibles du coefficient de corrélation phénotypique. De faibles valeurs du coefficient de corrélation ont également été observées pour les caractères faiblement héritables dans l'expérience de Singh et al. (1984).

Dans les quelques expériences permettant d'estimer ce paramètre sur le rendement en grain du maïs (Compton et al., 1965; Moll \& Stuber, 1971; Singh et al., 1984), les estimations du coefficient de corrélation génétique sont toutes

\begin{tabular}{|c|c|c|c|c|c|c|c|c|}
\hline & $M S$ & $R S$ & $R F$ & $H T$ & $H E$ & LOF & $L A F$ & FLO \\
\hline \multicolumn{9}{|l|}{ cycle $C_{0}$} \\
\hline cornée & $0,58^{*}$ & 0,10 & $0,57^{*}$ & $0,60^{*}$ & $0,57^{\star}$ & 0,13 & $0,61^{*}$ & $0,55^{*}$ \\
\hline dentée & $0,49^{*}$ & 0,32 & $0,75^{*}$ & $0,73^{*}$ & $0,55^{*}$ & $0,77^{*}$ & $-0,14$ & $0,70^{*}$ \\
\hline \multicolumn{9}{|l|}{ cycle $C_{1}$} \\
\hline cornée & $0,50^{*}$ & 0,10 & 0,28 & 0,62 * & $0,57^{*}$ & 0,52 * & $0,47^{*}$ & 0,39 * \\
\hline dentée & $0,89^{*}$ & $0,55^{*}$ & $0,83^{*}$ & $0,67^{*}$ & $0,70^{\star}$ & $0,70^{*}$ & $0,65^{*}$ & $0,76^{*}$ \\
\hline
\end{tabular}
positives et varient de 0,1 à 1,0 , selon les popu-

Tableau VII. Corrélations phénotypiques entre les effets moyens des plantes mâles estimés en croisement intra et interpopulation.

\footnotetext{
" : significatif à $5 \%$; l'explicitation de l'abréviation des caractères est sous le Tableau III.
} 
lations étudiées. L'insuffisance d'information sur le passé sélectif des populations et l'imprécision de l'estimation ne permettent pas de discuter plus en détail de ce paramètre.

\section{CONCLUSION}

La majorité des caractères observés dans cette expérience a une variabilité génétique de nature essentiellement additive dans les 2 populations et pour les 2 types de descendances. Ces caractères présentent également une corrélation élevée entre les effets des plantes mâles révélés en croisement intra- et interpopulation. Pour ces populations et au départ d'un schéma de sélection, l'organisation de la variabilité génétique ne semble pas très différente dans les descendances intra- et interpopulation. Par ailleurs, les populations expriment une assez forte dépression de consanguinité : chute de 15 à $20 \%$ pour le rendement et la hauteur entre $S_{0}$ et $S_{1}$ (Gouesnard, 1988). La coexistence d'une forte variance d'additivité et d'une dépression de consanguinité a été soulignée chez la luzerne et d'autres espèces fourragères allogames (Gallais, 1984). Cet auteur suggère que l'organisation de la variabilité génétique soit différente en croisement (complémentation entre linkats homologues) et en consanguinité (pas de complémentation).

De la synthèse bibliographique réalisée sur le maïs et de l'expérience interprétée, il ressort que le rendement est un caractère pour lequel :

- la variance de dominance apparaît assez élevée,

- les différences entre les variances d'additivité estimées en croisement intra- et interpopulation sont les plus fortes,

- le coefficient de corrélation entre les effets moyens des plantes mâles des deux types de descendances est le plus faible.

Le rendement serait gouverné par des effets d'additivité et de dominance : outre les problèmes de précision liés à ce caractère, la faible corrélation entre les effets moyens des plantes mâles s'expliquerait par l'importance relative des effets de dominance. Ce résultat est cohérent avec ceux de la revue bibliographique de Brun (1985), réalisée sur différentes espèces animales, où il est montré une liaison entre le niveau d'hétérosis (et d'héritabilité) d'un caractère et l'expression d'une interaction entre génotypes et la population des partenaires (considérée ici comme population testeur).

Les résultats de cette étude ont souligné l'importance des effets additifs dans la variation génétique totale pour la majorité des caractères et, par voie de conséquence, une corrélation élevée entre les effets des plantes mâles estimés en croisement intra- et interpopulation. Pour le matériel considéré dans cette étude, il est probable que la sélection récurrente réciproque améliorerait corrélativement les valeurs propres des populations et inversement que la sélection intrapopulation améliorerait la valeur en croisement des 2 populations. Cette conclusion est en accord avec celle de la synthèse de nombreuses expériences de sélection chez le maïs réalisée par Hallauer \& Miranda (1981, p 238). Dans ce matériel, le choix de la population testeur n'apparaît pas primordial. Les méthodes de sélection interpopulation et intrapopulation semblent pouvoir être choisies indifféremment. Le choix de la population testeur pourra se faire notamment sur des critères liés aux contraintes de mise en œuvre ou de gestion de la variabilité génétique. La sélection récurrente réciproque peut présenter l'avantage, en améliorant simultanément 2 populations l'une par rapport à l'autre, de limiter globalement les risques de dérive génétique.

\section{RÉFÉRENCES}

Bridges W.C. \& Knapp S.J. (1987) Probabilities of negative estimates of genetic variances. Theor. Appl. Genet. 74, 269-274

Brun J.M. (1982) Interactions géniteur $\times$ population des partenaires. I. Définition d'indicateurs. Ann. Génét. Sél. Anim. 14, 463-480

Brun J.M. (1985) Interactions géniteur $x$ population des partenaires. III. Synthèse bibliographique. Génét. Sél. Evol. 17, 561-578

Compton W.A., Gardner C.O. \& Lonnquist J.H. (1965) Genetic variability in two open-pollinated varieties of corn (Zea mays L.) and their $F_{1}$ progenies. Crop Sci. 5, 505-508

Darrah L.L. \& Hallauer A.R. (1972) Genetic effects estimated from generation mean in four diallel sets of maize inbreds. Crop Sci. 12, 615-621

Falconer D.S. (1960) Introduction to Quantitative Genetics. Oliver et Boyd. Edinburg and London

Gallais A. (1978a) Quelques réflexions sur les modèles actuels de la génétique quantitative et sur leur place en amélioration des plantes. Ann. Amélior. Plant. 28, 141-148

Gallais A. (1978b) Amélioration des populations, méthodes de sélection et création de variétés. III. Bases théoriques pour l'étude de la sélection récurrente réciproque. Ann. Amélior. Plant. 28, 637-666

Gallais A. (1984) An analysis of heterosis versus inbreeding effects with an autotetraploid cross-fertilized plant : Medicago sativa L. Genetics 106, 123-127

Gallais A., Vincourt P. \& Bertholleau J.C. (1983) Etude des critères de sélection chez le maîs fourrage : héritabilités, corrélations génétiques et réponse attendue à la sélection. Agronomie 3, 751-760

Goodrich C.L., Stuber R.E. \& Compton W.A. (1975) Average gene frequency estimates in two open-poliinated cultivars of corn. Crop Sci. 15, 746-749 
Gouesnard B. (1988) Variabilité génétique dans deux populations de maïs menées en sélection récurrente réciproque. Thèse de docteur de l'Institut National Agronomique, Paris-Grignon, $68 \mathrm{pp}$

Griffing B. (1962) Prediction formulae for general combining ability selection methods utilizing one or two random-mating populations. Aust. J. Biol. Sci. 15, 650665

Hallauer A.R. \& Miranda J.B. (1981) Quantitative Genetics in Maize Breeding. lowa State University Press, Ames

Hebert Y. (1986) Expression de la variabilité du phénomène d'hétérosis au cours de la vie de la plante chez le maïs. Thèse de docteur ingénieur, Rennes, $154 \mathrm{pp}$

Kempthorne O. (1957) An Introduction to Genetic Statistics. John Wiley, New York, 545 pp

Lindsey M.F., Lonnquist J.H. \& Gardner C.O. (1962) Estimates of genetic variance in open-pollinated varieties of cornbelt corn. Crop Sci. 2, 105-108

Melchinger A.E., Geiger H.H. \& Schnell F.W. (1985) Reciprocal differences in single-cross hybrids and their F2 and backcross progenies in maize. Maydica 30, 395-405

Melchinger A.E., Geiger H.H. \& Schnell, F.W. (1986) Epistasis in maize (Zea mays L.). 2. Genetic effects in crosses among early flint and dent inbred determined by three methods. Theor. Appl. Genet. 72, 231-239

Miranda-Filho J.B. \& Paterniani E. (1983) Intra- and interpopulation genetic parameter estimates and impli- cations in selection. Revista Brasileira de Genetica 6 , 15-27. (In: Plant Breeding Abstracts (1984), 2593)

Moll R.H. \& Stuber C.W. (1971) Comparison of response to alternative selection procedures initiated with two populations of maize (Zea mays L.). Crop Sci. 11, 706-711

Moreno-Gonzalez J. (1988) Diallel crossing system in sets of flint and dent inbred lines of maize (Zea mays L.). Maydica 33, 37-49

Moreno-Gonzalez J. \& Dudley J.W. (1981) Epistasis in related and unrelated maize hybrids determined by three methods. Crop Sci. 21, 644-651

Obilana A.T., Hallauer A.R. \& Smith O.S. (1979) Estimated genetic variability in a maize interpopulation. $J$. Hered. 70, 127-132

Reeder L.R., Hallauer A.R. \& Lamkey K.R. (1987) Estimation of genetic variability in two maize populations. J. Hered. 78, 372-376

Robinson H.F., Khalil A., Comstock R.E. \& Cockerham C.C. (1958) Joint interpretation of heterosis and genetic variances in two open-pollinated varieties of corn and their cross. Genetics 43, 868-877

Scheffé H. (1959) The Analysis of Variance. John Wiley \& Sons, Inc. New York, 477 pp

Singh H., Khehra A.S. \& Dhillon B.S. (1984) Estimates of gene frequencies in two heterotic maize populations. Theor. Appl. Genet. 69, 179-186

Singh H., Khehra A.S. \& Dhillon B.S. (1985) Genetic architecture of two heterotic of maize. Maydica 30 , 31-36 